\title{
The Phospholipid Flippase in Isolated Hog Gastric Vesicles : Evidence for Translocation of Endogenous Glycerophospholipids
}

\author{
Magotoshi Morii, Takanari Murata, Hidehiro Suzuki, \\ and Noriaki Takeguchi
}

Faculty of Pharmaceutical Sciences, Toyama Medical and Pharmaceutical University, 2630 Sugitani, Toyama 930-0194, Japan

\begin{abstract}
The gastric flippase has been found recently in isolated hog gastric vesicles. We found here that the flippase ATP-dependently translocates endogenous phosphatidylcholine, phosphatidylethanolamine and phosphatidylserine from the outer (cytosolic) to inner (luminal) leaflet of the vesicle membrane bilayer or to the vesicle interior. In the experiments, vesicles were incubated in the presence and absence of ATP, and change in the distribution of endogenous phospholipids between the outer and inner leaflets was determined. Furthermore, the vesicle diameter was measured by a quasi-elastic laser light scattering method. The diameter significantly decreased during the translocation, indicating that the glycerophospholipids are translocated into the vesicle interior. The decrease in the size depended on ATP hydrolysis and was inhibited by flippase inhibitors such as 2-methyl-8-(phenylmethoxy) imidazo-[1,2-a]pyridine-3-acetonitrile (SCH 28080) and 4,4'-diisothiocyanostilbene-2,2'-disulfonic acid (DIDS). The gastric flippase, which moves into the apical membrane of the parietal cell upon stimulation, may be a candidate that provides the cytoprotective hydrophobic barrier of adsorbed monolayer on the apical membrane covering the phospholipid bilayer and the luminal surface of transmembrane proteins.
\end{abstract}

Keywords : gastric flippase / proton pump / cytoprotection / phosphatidylcholine / phosphatidylserine / gastric acid

\section{Introduction}

The gastric parietal cell has intracellular lumen surrounded by the canalicular membrane. Gastric acid is initially secreted into the lumen by gastric proton pump, $\mathrm{H}^{+}, \mathrm{K}^{+}$-ATPase, which is present in the canalicular membrane of the parietal cell. The canalicular membrane must have a cytoprotective barrier against the very strong acid. One of the most plausible mechanisms for the barrier may be an adsorbed monolayer on the apical membrane, forming the tri-layer and rendering the luminal surface hydrophobic (Hills, 1993 and 1996; Bernhard et al. 1995). We found the gastric flippase in isolated gastric vesicles that also contain the gastric proton pump (Suzuki et al. 1997). The flippase translocates ATP-dependently fluorescent analogues of phosphatidylcholine (PC), phosphatidylethanolamine (PE) and phosphatidylserine (PS) from the outer (cytosolic side) to inner leaflet (luminal side) of the vesicle bilayer or further into the vesicle interior (Suzuki et al. 1997). If this flippase secretes phospho- 
lipids into the vesicle interior (luminal space), the flippase is able to engage in formation of the hydrophobic surface lining.

Therefore, we studied here 1) whether endogenous phospholipids of gastric vesicles are translocated by the flippase, 2) whether the direction of translocation of endogenous phospholipids is the same as that had been reported by Suzuki et al. (1997) using the fluorescent probes which were incorporated almost only in the outer leaflet of vesicle bilayer, producing the overwhelming mass gradient of the probe from the outer to the inner leaflet, and 3 ) whether the flippase transports these glycerophospholipids from the outer (cytosolic) leaflet of the bilayer to the vesicle interior (luminal space).

\section{Experimental}

\section{1 Materials and solutions}

Phospholipase C from Bacillus cereus (grade I , sphingomyelinase $<0.05 \%$ ) was obtained from Boehringer Mannheim (Mannheim, Germany). Sphingomyelinase from Bacillus cereus (phospholipase $\mathrm{C}<1 \%$ ) and tricyclodecan-9-ylxanthogenate (D609, a phospholipase C inhibitor (Kiss \& Tomono, 1995)) were obtained from Sigma (Tokyo, Japan). 2-Methyl-8-(phenylmethoxy)imidazo-[1,2-a]pyridine-3-acetonitrile (SCH 28080) was obtained from ScheringPlough Co (Bloomfield, NJ, U.S.A.). 4,4'-diisothiocyanostilbene-2,2'-disulfonic acid (DIDS) was obtained from Dojin Co. (Kumamoto, Japan). Gastric vesicles (GI fraction) were prepared from hog gastric mucosae that were obtained from a local slaughterhouse as described elsewhere (Takeguchi et al., 1983). Vesicles were stored below $-80{ }^{\circ} \mathrm{C}$ until use.

For phospholipid transport study, typically, 1 $\mathrm{m} l$ of solution containing $100 \mu \mathrm{g} / \mathrm{m} l$ tightly- sealed gastric vesicles, $130 \mathrm{mM} \mathrm{NaCl}, 4 \mathrm{mM}$ $\mathrm{MgCl}_{2}, 3 \mu \mathrm{g} / \mathrm{ml}$ creatine kinase, $2 \mathrm{mM}$ creatine phosphate, and $40 \mathrm{mM}$ Tris $/ \mathrm{HCl}$ (pH 7.40) was incubated at $37{ }^{\circ} \mathrm{C}$ for $60 \mathrm{~min}$ in the presence or absence of $2 \mathrm{mM}$ ATP, where creatine kinase and creatine phosphate were added to keep a constant ATP concentration.

\section{2 Determination of the distribution} of endogenous phospholipids in outer and inner leaflets of the gastric vesicle bilayer

To degrade glycerophospholipids in the outer layer of the vesicle bilayer, the vesicle solution was incubated, unless indicated, for $20 \mathrm{~min}$ at $37^{\circ} \mathrm{C}$ in the presence of 4 units of phospholipase C. This method was previously applied for the present gastric vesicle system and its validity was established (Olaisson et al., 1985). For degradation of sphingomyelin, 0.5 unit of sphingomyelinase plus $100 \mu \mathrm{g} / \mathrm{ml}$ of D609 were used. D609 was used to inhibit phospholipase C that was present in the reagent of sphingomyelinase as a contaminant. The reaction was terminated by the addition of $3.75 \mathrm{ml}$ of chloroformmethanol mixture $(1: 2(\mathrm{v} / \mathrm{v}))$ to the $1 \mathrm{~m} l$ solution. This method did not distinguish whether the remaining phospholipids after the lipase treatment existed in the inner leaflet of the vesicle bilayer or in the vesicle interior.

\section{3 Phospholipid analysis}

Phospholipid was extracted from the above reaction mixture as described elsewhere (Bligh $\&$ Dyer, 1959). The mixture containing chloroform and methanol was shaken for $2 \mathrm{~min}$ and stood for $10 \mathrm{~min}$ at room temperature. Then, $1.25 \mathrm{~m} l$ of chloroform and $1.25 \mathrm{~m} l$ of distilled water were added to the mixture and shaken for $30 \mathrm{~s}$. After centrifugation $(1,200 \times \mathrm{g})$ for $5 \mathrm{~min}$, the upper layer was removed carefully and discarded. To remove aqueous phosphorus from 


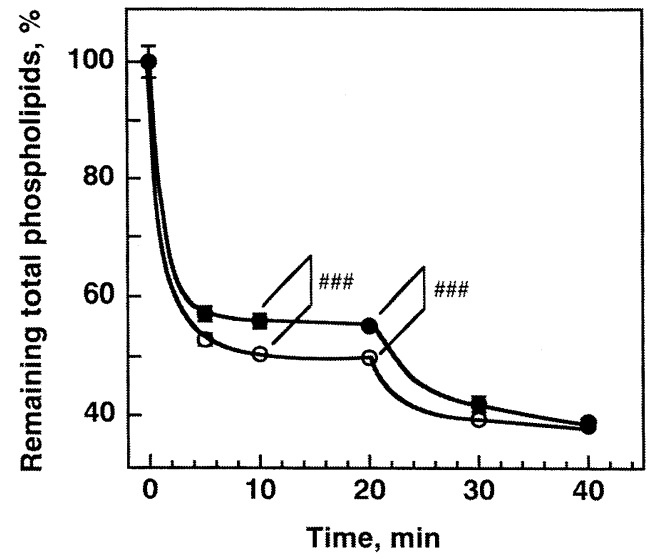

Fig. 1 Degradation of the gastric vesicle phospholipids with phospholipase $\mathrm{C}$.

Vesicles at $100 \mu \mathrm{g} / \mathrm{m} l$ were pre-incubated for $60 \mathrm{~min}$ at $37^{\circ} \mathrm{C}$ in the presence (O) and absence $(\bigcirc)$ of $2 \mathrm{mM}$ ATP, which was followed by another incubation with $4 \mathrm{unit} / \mathrm{m} l$ of phospholipase $\mathrm{C}$ for indicated various times. After $20 \mathrm{~min}$ incubation with phospholipase C, $5 \mathrm{mM}$ sodium cholate was added. The total phospholipids were determined, and expressed as \% of the initial total amount measured after $60 \mathrm{~min}$ pre-incubation in the absence of ATP. The data present means \pm S.E.M. of 3-4 experiments. $\# \# \# p<0.001$ (Dunnett's correction).

the lower layer, $2 \mathrm{~m} l$ of chloroform-methanolwater $(3: 48: 47(\mathrm{v} / \mathrm{v}))$ was added to the lower layer, and then the mixture was shaken and centrifuged. The resulting upper layer was discarded. This removal was repeated three times. The final lower layer was collected in a tube and organic solvent was evaporated under nitrogen gas stream. The phospholipid was dissolved into $20 \mu l$ of chloroform, spotted on a thin layer chromatography (TLC) plate (silica gel 60, $20 \times 20 \mathrm{~cm}$, Merck) and developed with methyl acetate / n-propanol / chloroform / methanol $/ 0.25 \% \mathrm{KCl}(25: 25: 25: 10: 9$ (v/v)). The developed phospholipids were colored and determined (Gustavsson, 1986).

The total phospholipid phosphorus was deter- mined as described elsewhere (Ames, 1966). The translocation activity of total glycerophospholipids was represented as the difference between the amounts of undegraded phospholipids incubated in the absence and presence of ATP.

\section{4 Determination of the vesicle diam- eter}

The vesicle diameter was determined by a quasi-elastic laser light scattering method (Morii et al., 1984) using LPA3000 system (Otsuka Electronics Co., Osaka, Japan) in a solution containing $100 \mu \mathrm{g} / \mathrm{m} l$ of vesicles, 130 $\mathrm{mM} \mathrm{NaCl}, 4 \mathrm{mM} \mathrm{MgCl}_{2}, 40 \mathrm{mM}$ Tris/ $\mathrm{HCl}(\mathrm{pH}$ 7.40) and $2 \mathrm{mM}$ ATP or 5'-adenylylimidodiphosphate (AMPPNP) at $25^{\circ} \mathrm{C}$.

\section{5 Statistical analysis}

Data are presented as means \pm S.E.M. Difference between two groups was analyzed by Student's $t$ test for unpaired data. Difference between more than two groups was analyzed by one-way analysis of variance (ANOVA), and correction for multiple comparisons was made by using Dunnett's multiple comparison. Statistical significance was assumed at $p<0.05$.

\section{Results}

\section{1 Distribution of phospholipids in the gastric vesicle bilayer}

Here, we used the vesicles that were pre-incubated for $60 \mathrm{~min}$ in the absence of ATP. The vesicles were found to contain $653 \pm 27 \mathrm{nmol}$ phospholipid / mg protein $(n=4)$. Fig. 1 shows the remaining undegraded total phospholipids in gastric vesicles as a function of the incubation time with phospholipase $\mathrm{C}$ that degrades glycerophospholipids (PC+PE+PS) (Fig. 1, open circles). The amount of undegraded total 
Table 1 The composition of major phospholipids in gastric vesicles.

\begin{tabular}{lc}
\hline & $\begin{array}{c}\text { Phospholipid composition } \\
\text { (mol \% of total phospholipids) }\end{array}$ \\
\hline PC & $20.9 \pm 1.1$ \\
PE & $23.8 \pm 1.6$ \\
PS & $11.8 \pm 0.9$ \\
SM & $38.2 \pm 0.5$ \\
\hline
\end{tabular}

Phospholipids (PC, phosphatidylcholine; PE, phosphatidylethanolamine ; PS, phosphatidylserine ; SM, sphingomyelin) were separated by the method of thin-layer chromatography, then colorized and quantified. The values present means \pm S.E.M. of 3-4 experiments.

phospholipids showed a plateau phase within 10 min, indicating that glycerophospholipids in the outer membrane was degraded in a short time (Olaisson et al., 1985). After $20 \mathrm{~min}$ incubation with phospholipase C, $5 \mathrm{mM}$ sodium cholate was added, which caused solubilization of the membrane resulting in a complete degradation of glycerophospholipids. From the values of the remaining total phospholipids at 0 and $40 \mathrm{~min}$, the initial amount of glycerophospholipids before degradation was found to constitute $61.8 \pm 0.5 \%$ (mean \pm S.E.M, $n=4)$ of the total phospholipids.

The composition (PC, PS and PE) was determined by the TLC analysis in 2 different preparations; vesicles incubated for $60 \mathrm{~min}$ in the absence of ATP (preparation 1) and vesicles incubated for $60 \mathrm{~min}$ in the absence of ATP followed by a 20 -min incubation with phospholipase $C$ (preparation 2). The analysis of preparation 1 gave that PC, PE and PS constituted 20.9, 23.8 and $11.8 \mathrm{~mol} \%$ of the total phospholipids (Table 1). Combining the results obtained for preparation 1 and 2 , the relative distribution of the inner leaflet for each phospholipid was calculated : PC in the inner leaflet (and/or the vesicle interior) constituted $12.5 \%$ of the total PC (Table 2) and the majority of PC was in the outer leaflet (87.5\%). Similarly, PE and PS in
Table 2 The transbilayer distribution of major phospholipids in gastric vesicles.

Each phospholipid distribution in the inner leaflet (\%) ; [inner leaflet/ (inner and outer leaflets) $] \times 100$

\begin{tabular}{lll} 
& - ATP & +ATP \\
\hline PC & $12.5 \pm 1.1$ & $15.6 \pm 0.7^{*}$ \\
PE & $15.2 \pm 0.5$ & $23.3 \pm 2.4^{*}$ \\
PS & $18.4 \pm 1.7$ & $22.0 \pm 2.6$ \\
SM & $66.2 \pm 1.3$ & $63.4 \pm 1.3$ \\
\hline
\end{tabular}

The relative distributions of $\mathrm{PC}, \mathrm{PE}, \mathrm{PS}$ and SM in the inner leaflet of the vesicle bilayer are expressed as \% of the sum of each phospholipid in the inner and outer leaflets. The distributions were measured after the incubation in the absence (middle column) and presence (right column) of ATP for $60 \mathrm{~min}$ at $37^{\circ} \mathrm{C}$. The values present means \pm S.E.M. of 3-4 experiments.

${ }^{*} p<0.05$ significantly different from the corresponding values measured in the absence of ATP for each phospholipids.

the inner leaflet constituted $15.2 \%$ and $18.4 \%$ of the total PE and PS, respectively.

We also determined the distribution of sphingomyelin (SM) in the vesicle membrane using sphingomyelinase and D609 in experiments similar to those shown in Fig. 1 for glycerophospholipids. It constituted $38 \mathrm{~mol} \%$ of the total phospholipids (Table 1) and $66 \%$ of SM was present in the inner leaflet and $34 \%$ was in the outer leaflet of the bilayer (Table 2). Fig. 2 shows the distribution of phospholipids in the inner and outer leaflets of the vesicle membrane bilayer expressed as \% of the total phospholipids.

\section{2 Translocation of endogenous glyc-} erophospholipids of gastric vesicles by the ATP-dependent flippase

Here, we used vesicles that were pre-incubated for $60 \mathrm{~min}$ in the presence of ATP. The amount of the total vesicle phospholipids incubated in the presence of ATP was the same as that incubated in the absence of ATP. Fig. 1 (closed circles) shows the remaining total phos- 


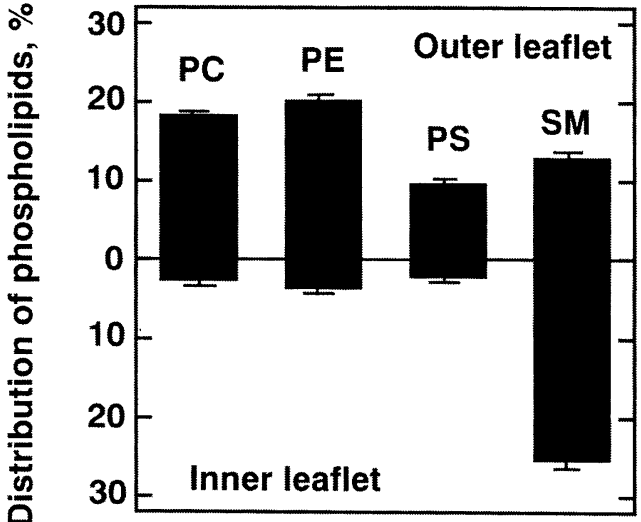

Fig. 2 The distribution of phospholipids in the inner and outer leaflets of the gastric vesicle bilayer.

Vesicles at $100 \mu \mathrm{g} / \mathrm{m} l$ were incubated for $60 \mathrm{~min}$ at $37^{\circ} \mathrm{C}$ in the absence of ATP, which was followed by another incubation with 4 units $/ \mathrm{m} l$ of phospholipase $\mathrm{C}$ or 0.5 $\mathrm{unit} / \mathrm{m} l$ of sphingomyelinase $+10 \mu \mathrm{g} / \mathrm{m} l$ of D609 for $20 \mathrm{~min}$. Phospholipids (PC, phosphatidylcholine ; PE, phosphatidylethanolamine ; PS, phosphatidylserine ; SM, sphingomyelin) were separated and quantified by a TLC method and the transbilayer distribution was expresses as mol \% of the total phospholipids. Data are means \pm S.E.M. of 3-4 experiments.

pholipids as a function of the degradation time with phospholipase C. At 10 and 20 min degradation times, the values obtained for the vesicle preparation incubated in the presence of ATP (closed circles) were significantly greater than those incubated in the absence of ATP (open circles), indicating that the translocation of phospholipids occurs in the presence of ATP from the outer to inner leaflet of the vesicle membrane (or further into the vesicle interior). Similar experiments using sphingomyelinase and D609 showed that SM was not significantly translocated from the outer to inner leaflet in the presence of ATP (Table 2).

Using the preparation incubated in the presence of ATP for 60 min followed by another incubation in the presence of phospholipase $\mathrm{C}$

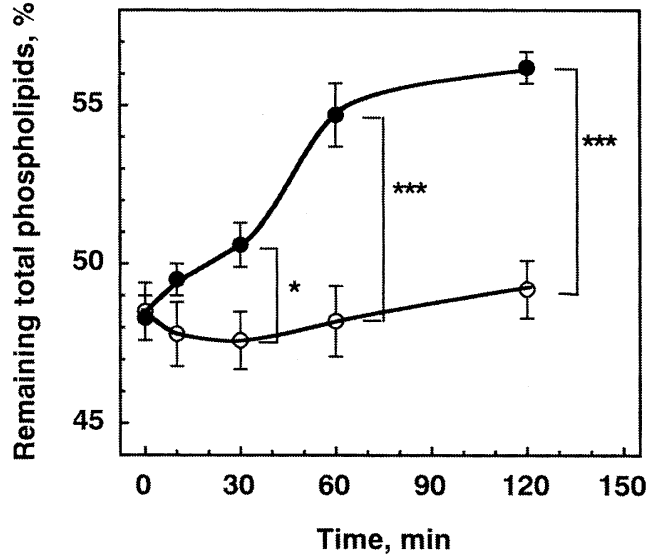

Fig. 3 The translocation of phospholipids from outer to inner leaflet of the vesicle membrane bilayer (or into the vesicle interior).

Vesicles were pre-incubated for the indicated various times in the presence (O) or absence (O) of $2 \mathrm{mM}$ ATP at $37^{\circ} \mathrm{C}$, which was followed by 20 -min incubation with phospholipase $\mathrm{C}$. Then the remaining total phospholipids were determined and expressed as \% of the initial amount of phospholipids measured after 60-min pre-incubation in the absence of ATP. $" p<0.05$ and ${ }^{* *} p<0.001$.

for $20 \mathrm{~min}$, we analyzed the composition of PC, $\mathrm{PE}$ and PS in the inner leaflet of the bilayer by the TLC method. The results are shown in Table 2. Comparison of the results obtained from the experiments in the absence of ATP (Table 2) shows that PC and PE were significantly translocated from the outer to inner leaflet (or further into the vesicle interior) in the presence of ATP. For example, $12.5 \%$ of PC was present in the inner leaflet and $87.5 \%$ of PC in the outer leaflet when incubated in the absence of ATP. The amount of the relative distribution of $\mathrm{PC}$ in the inner leaflet significantly increased to $15.6 \%$ when incubated in the presence of ATP. The present results indicate that the flippase shows the low selectivity between glycerophospholipids (PC, PE and maybe PS).

Fig. 3 shows the remaining total phospho- 


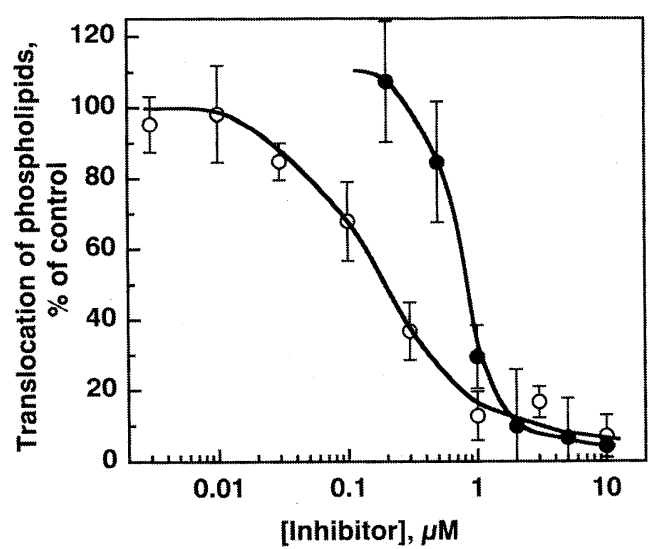

Fig. 4 Effect of inhibitors on the ATP-dependent translocation of glycerophospholipids in gastric vesicles.

Vesicles were pre-incubated in the absence and presence of ATP for $60 \mathrm{~min}$ at $37{ }^{\circ} \mathrm{C}$ together with various concentrations of flippase inhibitors $\mathrm{SCH} 28080$ $(\bigcirc)$ or DIDS (O), which was followed by another 20-min incubation with $4 \mathrm{unit} / \mathrm{m} l$ of phospholipase $\mathrm{C}$. The difference of the remaining total phospholipids between the samples incubated in the presence and absence of ATP is expressed as mol\% of control that is the amount obtained from the experiment without the inhibitors.

lipids in the gastric vesicles as a function of the pre-incubation time in the absence and presence of ATP, which was followed by 20 -min incubation with phospholipase $\mathrm{C}$. The presence of ATP in the incubation solution significantly increased the amount of glycerophospholipids in the inner leaflet of the vesicle membrane and the ATP-dependent increase depended on the incubation time (Fig. 3, closed circles). The amounts of this ATP dependent increase at 60 min depended on vesicle preparations ranging from $4.3 \%$ to $9.1 \%$ of the total phospholipids, its average being $6.1 \pm 0.4 \%$ of total phospholipids, $\mathrm{n}=38$ in 4 preparations.

Fig. 4 shows concentration-dependent effects of inhibitors, SCH 28080 and DIDS, on this ATPdependent glycerophospholipids transport. The

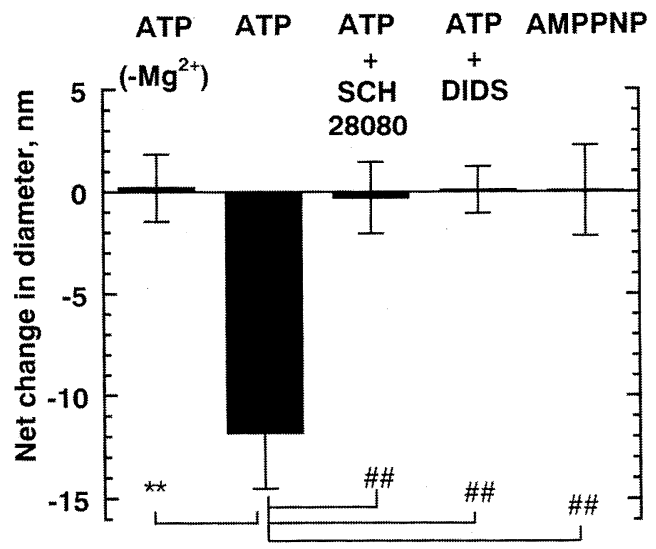

Fig. 5 Change in the vesicle diameter during incubation under different conditions.

The mean diameter of vesicles was measured at $25^{\circ} \mathrm{C}$ before and after the 60 -min incubation in a solution containing $100 \mu \mathrm{g} /$ $\mathrm{m} l$ of protein, $130 \mathrm{mM} \mathrm{NaCl}, 4 \mathrm{mM} \mathrm{MgCl}$ and $40 \mathrm{mM}$ Tris $/ \mathrm{HCl}(\mathrm{pH} 7.40)$ in the presence of $2 \mathrm{mM}$ ATP or AMPPNP. When indicated, $\mathrm{MgCl}_{2}$ was deleted from the solution, or $10 \mu \mathrm{M} \mathrm{SCH} 28080$ or 10 $\mu \mathrm{M}$ DIDS was supplemented. The mean diameter of vesicles before the incubation was $221 \pm 3 \mathrm{~nm} \quad(\mathrm{n}=6)$. The difference between the values of the diameter measured before and after 60-min incubation was defined as the net change in the diameter, and negative values show decrease in the size. The data are from 5 experiments.

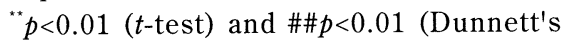
correction).

values of $\mathrm{IC}_{50}$ for SCH 28080 and DIDS were 0.14 and $0.78 \mu \mathrm{M}$, respectively, which are comparable with the previous results obtained using a fluorescent probe of $\mathrm{PC}, \mathrm{IC}_{50}$ being 0.14 and 3 $\mu$ M, respectively (Suzuki et al., 1997).

\section{3 Change in the vesicle diameter} during the ATP-dependent phospholipid translocation

The size of present hog gastric vesicles was previously studied by us (Morii et al., 1984) and the size distribution was found to be extremely homogenous. The averaged size was $221 \pm 3$ 
$\mathrm{nm}(\mathrm{n}=6)$. Fig. 5 shows that the vesicle size does not change after the 60-min incubation in the presence of ATP when $\mathrm{Mg}^{2+}$ is removed from the solution. But, it significantly decreased in the presence of $\mathrm{Mg}^{2+}$ and ATP by about $5 \%$. The decrease was inhibited by $10 \mu \mathrm{M} \mathrm{SCH}$ 28080 and $10 \mu \mathrm{M}$ DIDS, indicating that the inhibition of the flippase diminishes the change in the diameter. When AMPPNP was used in place of ATP, the change in the diameter did not occur. In these experiments, changes in the diameter were measured in the solution devoid of $\mathrm{KCl}$ to avoid activation of $\mathrm{H}^{+}, \mathrm{K}^{+}$-ATPase. To further avoid possible effect of ion movements, we added $10 \mu \mathrm{M}$ monensin in the solution. The addition did not affect the initial vesicle size, and the incubation in the presence of ATP for 60 min decreased the size irrespective of the presence of monensin, indicating that the size decreases found in the present study were not due to the ion movements (data not shown).

\section{Discussion}

Major fractions of the total PC, PE and PS are present in the outer leaflet of the gastric vesicle bilayer (Fig. 2), which is in agreement with the previous report (Olaisson et al., 1985). The present results have demonstrated that the flippase in the vesicles translocates endogenous glycerophospholipids from the outer to inner leaflet of the vesicle bilayer or further into the vesicle interior. This gastric flippase transports both $\mathrm{PC}$ and PE (and possibly PS, although this is not statistically significant), which is different from so far known ATP-dependent flippases. For example, aminophospholipid flippase (translocase) exists in various cell types and selectively translocates PS and PE but not PC from the cell surface to cytoplasmic leaflet, forming asymmetric distribution of membrane phospholipids (Auland et al., 1996 ; Tang et al., 1996). During blood coagulation or cell apoptosis, PS appears on cell surface and its exposed PS acts as pro-coagulation fields or phagocytosis recognition signals (Fadok et al., 1992 ; Bratton et al., 1997). As another example, human MDR2 (=MDR3)/P-gp translocates only PC and is involved in PC secretion into bile (Ruetz \& Gros, 1995 ; van Helvoort et al., 1996).

Another important finding in this study is that the size of gastric vesicles significantly decreases in the process of ATP-dependent translocation of glycerophospholipids (Fig. 5). If the transported phospholipids from the outer leaflet to the inner leaflet were not secreted in the vesicle interior, the vesicle size would not change. Therefore, the present results show that the glycerophospholipids are translocated into the vesicle interior. The energy of ATP may be used for the secretion of phospholipids.

In this study we used isolated gastric vesicles that originated gastric tubulovesicles, which contain the proton pump and the flippase. The tubulovesicles fuse with the apical membrane of the parietal cell upon stimulation of acid secretion by histamine. Thus, the gastric flippase in the apical membrane may be able to secrete glycerophospholipids. As previously suggested by others (Bernhard et al., 1995 ; Hills, 1996), the secreted glycerophospholipids may provide the hydrophobic barrier of adsorbed monolayer on the apical membrane covering the phospholipid bilayer and the luminal surface of transmembrane proteins against the secreted hydrogen ions.

\section{Acknowledgements}

This work was supported in part by Grants-in-Aid from the Ministry of Education, Science, Sports and Culture of Japan to NT. 


\section{References}

Ames, B. N. Assay of inorganic phosphate, total phosphate and phosphatase. Methods in Enzymology 8, 115-118 (1966)

Auland, M. E., Roufogalis, B. D., Devaux, P. F. \& ZACHOwsKI, A. Reconstitution of ATP-dependent aminophospholipid translocation in proteoliposomes. Proceedings of the National Academy of Sciences of the United States of America 91, 1093810942 (1994)

Bernhard, W., Postle, A. D., Linck, M. \& Sewing, K. F. Composition of phospholipid classes and phosphatidylcholine molecular species of gastric mucosa and mucus. Biochimica et Biophysica Acta 1255, 99-104 (1995)

BLIGH, E. G. \& DYER, W. J. A rapid method of total lipid extraction and purification. Canadian Journal of Biochemistry and Physiology 37, 911-917 (1959)

Bratton, D. L., FAdOK, V. A., Richter, D. A., KaILEY, J. M., Guthrie, L. A. \& Henson, P. M. Appearance of phosphatidylserine on apoptotic cells requires calcium-mediated nonspecific flip-flop and is enhanced by loss of the aminophospholipid translocase. Journal of Biological Chemistry 272, 26159-26165 (1997)

Fadok, V. A., Voelker, D. R., Campbell, P. A., Cohen, J. J., Bratton, D. L. \& Henson, P. M. Exposure of phosphatidylserine on the surface of apoptotic lymphocytes triggers specific recognition and removal by macrophages. Journal of Immunology 148, 2207-2216 (1992)

Gustavsson, L. Densitometric quantification of individual phospholipids. Improvement and evaluation of a method using molybdenum blue reagent for detection. Journal of Chromatography 375, 255-266 (1986)

HILls, B. A. Gastric mucosal barrier: evidence for Helicobacter pylori ingesting gastric surfactant and deriving protection from it. Gut 34, 588-593 (1993)

HILls, B. A. Gastric surfactant and the hydropho- bic mucosal barrier. Gut 39, 621-624 (1996)

KIss, Z. \& Tomono, M. Compound D609 inhibits phorbol ester-stimulated phospholipase D activity and phospholipase C-mediated phosphatidylethanolamine hydrolysis. Biochimica et Biophysica Acta 1259, 105-108 (1995)

Mori, M., Ishimura, N. \& TAKeguchi, N. Quasielastic light-scattering studies of conformational states of the H,K-ATPase. Intervesicular aggregation of gastric vesicles by disulfide cross-linking. Biochemistry 23, 1984 6816-6821 (1984)

Olaisson, H., Mardh, S. \& ARvidson, G. Phospholipid organization in H,K-ATPase-containing membranes from pig gastric mucosa. Journal of Biological Chemistry 20, 11262-11267 (1985)

RuETZ, S. \& Gros, P. Enhancement of Mdr2-mediated phosphatidylcholine translocation by the bile salt taurocholate. Journal of Biological Chemistry 270, 25388-25395 (1995)

Suzuki, H., Kamakura, M., Morit, M. \& TAKEGUCHI, N. The phospholipid flippase activity of gastric vesicles. Journal of Biological Chemistry 272, 10429-10434 (1997)

Takeguchi, N., Joshima, R., Inoue, Y., KashiwaGURA, T. \& MORII, M. Effects of $\mathrm{Cu}^{2+}-o$-phenanthroline on gastric $\left(\mathrm{H}^{+}+\mathrm{K}^{+}\right)$-ATPase. Evidence for opening of a closed anion channel conductance by S-S cross-linkings. Journal of Biological Chemistry 258, 3094-3098 (1983)

Tang, X., Halleck, M. S., Schlegel, R. A. \& Williamson, P. A subfamily of P-type ATPases with aminophospholipid transporting activity. Science 272, 1495-1497 (1996)

van Helvoort, A., Smith, A. J., Sprong, H., Fritzsche, I., Schinkel, A. H., Borst, P. \& VAN Meer, G. MDR1 P-glycoprotein is a lipid translocase of broad specificity, while MDR3 P-glycoprotein specifically translocates phosphatidylcholine. Cell 87, 507-517 (1996)

(受付 2000 年 5 月 16 日 修正稿受付 6 月 14 日 掲載決定 6 月 19 日） 